\title{
Effects of dry needling in gluteus medius muscle in individuals with chronic ankle instability: a randomized single-blinded controlled clinical trial
}

\section{Type}

Research paper

\section{Keywords}

pain, dynamic balance, dry needling, ankle inestability

\begin{abstract}
Introduction

Background: Lateral ankle sprain (LAS) is the most common musculoskeletal injuries in sport and general population. The goal of the present study was to observe the effectiveness of dry needling (DN) in gluteus medius muscle in patients with chronic ankle instability (CAI).
\end{abstract}

\section{Material and methods}

A two-arm (1:1), single-blinded (participants), randomized clinical trial was performed in 40 subjects with chronic ankle instability and were divided in two groups: intervention group who received one session of dry needling in the most hyperalgesic gluteus medius myofascial trigger point (MTrP), $(n=$ 20 ) and control group $(n=20)$. Dynamic balance, pain intensity, pain pressure threshold (PPT) and ankle dorsiflexion range of motion (ROM) were assessed at baseline, post-intervention and a 1-week follow up.

\section{Results}

The experimental group reported significant differences with respect to the control group for the anterior and medial dynamic balance $(p=.001)$, PPT -ATL $(p=.002)$ and ankle dorsiflexion ROM ( $p$ $=.001)$.

\section{Conclusions}

The findings of the present study suggested that the DN in the most hyperalgesic MTrP of the Gmed muscle may increase the anterior and medial dynamic balance, ankle ROM and PPT-ATL at shortterm in individuals with CAI. Pain intensity benefits were reported in both groups. Future studies should consider DN as a possible intervention in conjunction with a physical therapy program for individuals with CAI. 
1 Effects of dry needling in gluteus medius muscle in individuals with chronic ankle

2

3

\section{Abstract}

5 Background: Lateral ankle sprain (LAS) is the most common musculoskeletal injuries in 6 sport and general population. The goal of the present study was to observe the 7 effectiveness of dry needling (DN) in gluteus medius muscle in patients with chronic 8 ankle instability (CAI). Methods: A two-arm (1:1), single-blinded (participants), randomized clinical trial was performed in 40 subjects with CAI and were divided in two groups: intervention group who received one session of $\mathrm{DN}$ in the most hyperalgesic gluteus medius myofascial trigger point $(M \operatorname{Tr}),(n=20)$ and control group $(n=20)$. Dynamic balance, pain intensity, pain pressure threshold (PPT) in the anterior talofibular ligament (ATL) and ankle dorsiflexion range of motion (ROM) were assessed at baseline, post-intervention and a 1-week follow up. Results: The experimental group reported significant differences with respect to the control group for the anterior and medial dynamic balance $(\mathrm{p}=.001)$, PPT -ATL $(\mathrm{p}=.002)$ and ankle dorsiflexion ROM $(\mathrm{p}=.001)$. Conclusion: The findings of the present study suggested that the DN in the most hyperalgesic MTrP of the Gmed muscle may increase the anterior and medial dynamic balance, ankle ROM and PPT-ATL at short-term in individuals with CAI. Pain intensity benefits were reported in both groups. Future studies should consider DN as a possible intervention in conjunction with a physical therapy program for individuals with CAI. 


\section{Introduction}

Lateral ankle sprain (LAS) is the most common musculoskeletal injuries in sport and general population.(1) In addition, the societal cost as high as $\$ 6.2$ billion annually.(2) Patients who suffer a LAS episode commonly report a decreasing in quality of life, foot and ankle osteoarthritis, a lack of lower limb functionality and thus, a decreasing in sports performance.(2) A 70\% of those individuals may develop chronic ankle instability (CAI), a disorder featured by subjective instability, recurrent episodes of "giving away" and sprains, loss of function, postural control, reflex function, mechanical and sensorimotor disturbances.(3) Moreover, Terrier et al.(4) reported that an altered proprioception, balance and motor control can be reported in patients with CAI. Several authors described that patients with CAI have been an altered gait kinematics, disturbances in ligament laxity, range of motion (ROM) alterations and synovial changes.(5)(6)(7) The summation of this symptoms may predispose an increase in the risk of recurrent LAS associated to a laterally deviated center of pressure and disturbances in balance during the gait movement.(8)(9)

It has been reported that patients with CAI showed an increase of peroneus longus (PL) activity prior to the initial ground contact.(10) In addition, in an unilateral drop jump patients with CAI reported a decreasing of the PL muscle activation compared with healthy individuals assessed by electromyography.(11) Hale et al.(12) argued that the existence of disturbances in both peripheral and centrally mechanisms could be developed after injury processes, which produces alterations in postural control after rehabilitation programs. Moreover, authors suggest that a balance program training on lower extremity could have benefits in the foot and ankle function and balance. Despite of most of the research about muscular motor control and patterns have been carried out in proximal ankle joints in subjects with CAI (e.g. decreasing muscle activation in muscles that surrounding the ankle, knee and hip),(13) Webster and Gribble(14) showed a decreasing of gluteus maximus (Gmax) muscle activity in patients with CAI during a single leg rotational squat work.

Postural control plays an important role for the development of an adequate muscular activation and a coordinated gait pattern. The Star Excursion Balance Test (SEBT) was defined as a reliable and valid tool to assess the dynamic postural control between individuals with and without ankle pathology.(15) In addition, patients with CAI showed a postural control deficit compared with healthy individuals measured by SEBT. Likewise, the Y-Balance test (YBT) was also defined a SEBT variation tool to assess the 
dynamic balance of the lower limb with excellent reliability values (intra-rater ICC $=0.85$ to 0.91 and inter-rater ICC $=0.91$ to 0.99$)(16)$ and it was employed in previous studies.(17)

Ayotte et al.(18) reported that an appropriate Gmax and gluteus medius (Gmed) function is necessary for maintaining postural stability during weight bearing activities. In this line, Jaber et al. compared the postural control and electromyographic (EMG) activity between individuals with and without CAI. The results showed that alterations in proximal and distal muscle activity have negative effects in quality of movement and postural control, which may lead long-term functional.(19) Thus, authors suggested that interventions and training programs focused in hip and ankle muscles could have benefits in the prevention and management in patients with CAI.

Myofascial trigger points (MTrPs) located at the Gmax and Gmed were described as a result of muscle weakness, biomechanical disturbances of the lower limbs or lumbar pathology.(20) MTrPs were defined as an "hyperirritable nodule in a taut band of skeletal muscle wihich is palpable and tender during the physical evaluation".(21) In addition, MTrPs were related with overuse, motor control disturbances, injuries and pain. Two classfications of MTrPs were defined: active MTrPs reproducing symptoms and referred pain and, latent MTrPs had no symptoms but can be palpable in a clinical examination. Regarding the diagnosis, active MTrPs performed a recognizable pain and local twitch response to a needle penetration.(20)

Dunning et al.(22) argued that dry needling (DN) have benefits on the management of the neuromusculoskeletal pain syndromes, such as the treatment of MTrPs. In addition, Salom et al.(23) reported that DN is an effective approach for the treatment of sensory and motor factors in MTrPs. Several authors showed the benefits of the DN in the pain management, for example $\mathrm{Hu}$ et al.(24) conducted a meta-analysis in patients with low back pain showing that DN was more effective than acupuncture for reducing pain intensity. In the same line, a systematic review and a meta-analysis carried out by Gattie et al.(25) reported that $\mathrm{DN}$ was superior to sham treatment for short- and mid-term follow ups for musculoskeletal pain conditions. In addition, DN performed in the sternocleidomastoid muscle have also shown an increase of the motor control of the cervical muscles for a 1-month follow up in individuals with neck pain(26). SánchezMila et al.(27) reported the benefits of DN added to a Bobath program in ROM, balance and also reducing the spasticity in patients who had suffered a stroke. 
102 Several authors reported that an improvement of the motor control and function of hip 103 muscles, such as Gmax and Gmed could be benefits on the lower limb stability. In 104 addition, for the treatment or prevention in patients who develop CAI. Thus, the aim of 105 the present study was to determine the effect of a DN intervention in the most 106 hyperalgesic latent MTrPs of the Gmed, mainly related to ankle ROM, dynamic balance 107 and the pressure pain threshold (PPT) of the anterior talofibular ligament (ATL) and 108 MTrPs of the Gmed muscle compared with a sham intervention in individuals with CAI.

109 We hypothesized that individuals receiving DN would exhibit greater improvements in 110 ankle ROM, balance and PPT than those patients receiving a sham intervention.

Methods

112 Design

113 A two-arm (1:1), single-blinded (participants), randomized clinical trial was performed 114 from September 2019 to January 2020 following the CONsolidated Standards of the 115 Reporting Trials criteria.(28)

\section{Ethical considerations}

117 Previously, the Clinical Research Ethics Committee of Hospital de la Princesa (Madrid, 118 Spain) approved the study and was registered in Clinicaltrials.gov (NCT04108390). All 119 the participants signed the informed consent form before the beginning of the study. 120 Moreover, all the participants respected and took into account the Helsinki Declaration 121 and ethical standards for human experimentation.

\section{Sample size calculation}

$123 \mathrm{G}^{*}$ Power software was employed for the sample size calculation by the difference between the intervention group and control group using the ATL-PPT $\left(\mathrm{kg} / \mathrm{cm}^{2}\right)$ variable of a pilot study $(\mathrm{n}=12)$ divided in two groups (mean $\pm \mathrm{SD}), 6$ subjects for the A group (intervention) $(4.42 \pm 0.25)$ and 6 subjects for the B group (control) $(4.22 \pm 0.21)$. For the sample size calculation, a power of 0.80 , an $\alpha$ error of 0.05 and effect size of 0.86 with 1 tailed hypothesis were employed. In conclusion, a sample of 36 was calculated. However, we could recruit sample of 40 individuals for this study.

A total sample of 40 subjects with CAI based on the position statement of the International Ankle Consortium(29) from a care center was recruited for the present study and divided in two groups: intervention group $(n=20)$ and control group $(n=20)$. (Figure 1). Inclusion criteria were as follows: history of at least 2 recurrent ankle sprains with inflammatory symptoms (e.g. pain, swelling), at least 2 episodes of "giving away" in the 
1366 months before study enrollment.(5) Subjects were excluded if they: had no history for

137 ankle sprain, history of vestibular disorders, lower limb surgeries in the previous 12 138 months (e.g. fractures or muscular tears), patients who received a physiotherapy, medical

139 o pharmacology treatment in the previous 3 months.(19)

\section{Randomization and blinding}

141 Before the intervention, the random process was developed with the free software system

142 randomization.org with 1:1 allocation radio and assigned the participants to the A group

143 (intervention) or B group (control). Therefore, patients for each group were do not known

144 which group they belong.

\section{Interventions}

146 Participants included in this study received 1 intervention with 3 evaluations: pre147 intervention, post-intervention and at 1 -week. We used the same needles $(0.32 \times 40 \mathrm{~mm})$

148 for both interventions. In addition, the interventions were performed by the same therapist 149 with more than 10 years of experience in DN technique for the management of MTrPs. 150 Before the needle application, the area was disinfected with skin antiseptic. Immediately after the intervention, the therapist applied pressure into the skin in order to prevent excessive bleeding with a cotton bud.

\section{Intervention group}

154 Only one session of DN to the intervention group on the ipsilateral Gmed muscle of the 155 CAI lower limb using Hong's "fast in" and "fast out" intervention with multiple rapid 156 needle insertion was applied following previous guidelines.(30)(31) Each participant 157 received the same DN treatment in the most hyperalgesic latent MTrP located on the 158 Gmed muscle.

\section{Control group}

160 Following Pecos et al.(32) guidelines, the control group received the same protocol on

161 the ipsilateral Gmed muscle of the CAI lower limb, but the needle was inserted $1.5 \mathrm{~cm}$ 162 medially from the MTrP (outside the MTrP location).

\section{Outcome measurements}

164 PPT was defined as the amount of pressure generated of the target point to be evaluated 165 until painful sensation appears.(33) For the present study, PPT was assessed from 0 to 10 $\mathrm{kg} / \mathrm{cm}^{2}$ with a mechanical algometer (FDK/FDN, Wagner Instruments, Greenwich, CT).

167 In addition, this algometer is reliable, sensitive and reproducible for latent $\mathrm{MTrP}$ 168 assessments.(34)(35)(36) The most hyperalgesic latent MTrP in the Gmed and the ATL 
were assessed. The evaluation procedure was performed by the mean of three repeated measurements with a $30-60$ s rest interval between evaluations.

The YBT consist of three lines attached to the floor in the anterior posteromedial and posterolateral directions. Following the Pliski et al.(16) procedure, the posterior lines were located 135 degrees from the anterior line with 45 degrees between the posterior lines. Before the test, individuals viewed an instructional video about the procedure in order to the familiarization process. Once the demonstration have been carried out, the subjects practiced six trials on each leg of the three directions prior the formal assessment.(16) The subjects were in standing barefoot at the center of the "Y" mark. Each participant should to maintain a single-leg stance of the target limb in order to reach the maximum distance in anterior, posterolateral and posteromedial directions. The subject's hands were placed on their hips and the stance heel should be remain in contact with the ground. If during the assessment any criteria were violated, the trial should be repeat. To calculate the normalized YBT values the individual's leg length was measured in supine position from the anterior superior iliac spine to the malleolus tibialis.(37) The distance was quantified in centimeters. For the normalization the following formula was applied dividing the mean reach distance by the individuals leg length and multiplying by $100 \%$.

Maximal ankle dorsiflexion ROM was evaluated using a standard manual goniometer and were defined as the distance of the toe from the wall maintaining the contact between wall and knee without lifting the heel. Thus, the individuals reach the final lunge position at maximal dorsiflexion and the goniometer was aligned with the mobile branch at the fibula and the stable branch aligned with the fifth metatarsal head.(38) For each measurement, the evaluator passively moved the ankle from a neutral baseline position to a dorsiflexion until a firm end-feel was bringing out.

All the outcome measurements were carried out by the same investigator (G.J.C).

\section{Statistical analysis}

SPSS 23.0 software (IBM SPSS Statistics, Armonk-NY; IBM-Corp) was employed for the statistical analysis. Kolmogorov-Smirnov test was used to assess normality data distribution. Student $t$ test was applied to test age, weight, height, BMI and Cumberland Ankle Instability Tool (CAIT) differences between groups. In order to check the basal values of the main variables the Student $t$ test was also employed. To assess the effects 
of intra-subjects (time) and inter-subject (treatment groups) values on the dependent variables, a two-way analysis of variance (ANOVA) for repeated measures was performed (considering the significance of the Greenhouse-Geisser correction when the Mauchly test rejected the sphericity). The Tukey post-hoc test was employed for multiple comparisons. Furthermore, the effect size was calculated by the Eta ${ }^{2}$ coefficient. For nonparametric data, Friedman test and Wilcoxon post-hoc analyses were employed for intrasubject comparisons. Moreover, Mann-Whitney $U$ test was employed for the comparisons between groups. The level of significance was set at $\mathrm{P}<0.05$ with an $\alpha$ error of $0.05(95 \%$ confidence interval) and a desired power of $80 \%$ ( $\beta$ error of 0.2$)$.

\section{Results}

211 Regarding the table 1, sociodemographic data did not show significant differences

$212(\mathrm{P}>$.05). Moreover, Student $t$ test for the main variables reported significant differences between groups for ATL-PPT $(\mathrm{P}=0.015)$ and VAS $(\mathrm{P}=0.002)$ baseline variables. The rest of variables did not show significant differences $(\mathrm{P}>.05)$ between the intervention and control group. Time interaction effects reported significant differences $(\mathrm{P}>.05)$ for

216 SEB anterior, SEB lateral, SEB medial, dorsiflexion ROM, ATL-PPT and Gmed PPT

217 variables. Significant differences were observed between groups for an increase of YBT anterior $(\mathrm{P}=.001)$, YBT medial $(\mathrm{P}=.001)$, dorsiflexion ROM $(\mathrm{P}=.001)$ and ATL-PPT $(\mathrm{P}=.002)$ in favor the intervention group with respect to the control group. In addition, no significant differences were obtained for Gmed PPT (P =.332) variable. (Table 2) In

221 addition, Tukey post-hoc analysis reported significant differences $(\mathrm{P}>.05)$ between baseline and post-intervention measurements for ATL-PPT, dorsiflexion ROM, YBT anterior, YBT lateral and YBT medial variables for the experimental group. Significant differences between baseline and 1-week for ATL-PPT, dorsiflexion ROM, YBT anterior, YBT lateral and YBT medial variables in the experimental group. (Figure 2) 
226 Regarding the pain intensity variable, Friedman test reported differences $(p=0.001)$ for the experimental group and the control group $(\mathrm{p}=0.043)$. Mann-Whitney $U$ test reported differences between groups $(\mathrm{p}=004)$ but non-significant differences $(\mathrm{P}>.05)$ were reported between groups at post-intervention and at 1-week follow up.

\section{Discussion}

231 To the authors' knowledge, this research study may be considered the first clinical trial showing benefits in balance, ankle dorsiflexion ROM and ATL- PPT in individuals with CAI who received a DN intervention in the most hyperalgesic MTrP at the Gmed muscle. In addition, pain intensity benefits were found in both groups. These findings were in accordance with previous studies who reported the benefits of DN in MTrP at the PPT variable in individuals with temporomandibular disorders,(39) chronic shoulder pain,(40) neck pain,(41) patients after knee arthroplasty,(42) or plantar heel pain.(43) Moreover, the results of the present study reported significant differences for the increase of the ATL-PPT immediately post-intervention and at 1-week follow up compared with the control group. Regarding the pain intensity at the experimental group, benefits were

241 shown immediately after the DN application and at 1-week follow up as well as the

242 control group. In a systematic review and meta-analysis conducted by Liu et al.(44) showed that DN intervention of MTrP may be recommended to relieve the pain intensity at short- and medium-term in individuals with low back pain, but the effects with larger follow-ups remains unclear. In addition, in a systematic review conducted by the same authors 3 years before authors reported that $\mathrm{DN}$ was effective to relieve the pain intensity

247 in subjects with low back pain at post-intervention and reported that further research is needed to improve the knowledge of the effectiveness with longer follow-ups in DN interventions. Considering the positive effects of a DN approach in the PPT, prior studies reported benefits at the increase in PPT levels in the masseter muscle in patients with 
temporomandibular disorders,(39) cervical spine (C7) in subjects with chronic neck pain with a DN treatment in the upper trapezius muscle,(45) or in individuals with unilateral shoulder impingement syndrome with a DN in the upper trapezius muscle.(46) Our results were related with prior studies that showed benefits in pain intensity and PPT in ATL at short-term with the application of DN in MTrP. A possible explanation for the effectiveness of the DN in pain variables could be related with the gait control mechanism for the speedily penetration of the needle into a MTrP might stimulate the afferent sensory fibers, which produce an inhibition in the dorsal horn of the spinal cord by blocking the pain afferences developed in the MTrP nociceptor.(47)

Several authors reported the effectiveness of the DN technique (isolated or combined with other therapies) in the ROM. For example, Onat et al.(48) found an increase of ROM after

262 the application of DN into the posterior paracervical muscle in individuals with mechanical neck pain. Mendiguita-Gómez et al.(49) included a DN treatment for the spastic shoulder muscles in individuals who had experienced a stroke and reported an increase of the ROM. The present study showed the effectiveness of a DN approach increasing the ankle dorsiflexion ROM, these findings could be related with the

267 improvement of the dynamic balance of the lower limb, which can help to increase the ankle mobility. In addition, the results reported by several authors about the ROM after a DN treatment did not match with what we found in our study. (50)(51) Therefore, further research is needed in order to a better understanding of the possible relationship between

271 the motor control and ROM.

272 Regarding the dynamic balance, MacKinnon and Winter argued that the gait in the frontal 273 plane is regulated by the subtalar and hip joint.(52) Thus, a deficit in the motor control at 274 the muscles involved with the hip kinematics would influence the gait in individuals with 275 and without pathology. In addition, Jun Son et al.(5) suggested that the ankle joint corrects 
small disturbances related with a deficient foot position, and the hip correct large

277 disturbances associated to a deficient foot position. Thus, the ankle and hip have to work

278 in synergy. When the foot adopts vulnerable positions or in an overcompensation ankle

279 kinematics (e.g. ankle sprains, forced inversion) the lower limb dynamic balance might

280 be corrected for an increase of muscle activity of the ankle evertors and hip abductors,

281 such the Gmed. Our results reported the effectiveness of a DN intervention in the most hyperalgesic MTrP located in the Gmed muscle in individuals with CAI for the increase in dynamic balance with respect to controls for the YBT anterior and medial variables. In addition, anterior, medial and lateral YBT variables reported differences between baseline and 1-week follow up in the experimental group. A possible explanation of these results could be an increase in the motor control of the targeted MTrP at the Gmed muscle and an improvement of the musculoskeletal pain conditions. Despite of in this study did not performed a electromyography analysis, several authors related the effectiveness of the MTrP DN with a muscle function improvement.(53)(54) The activation of 6 lower extremity muscles was reduced in individuals with $\mathrm{CAI}$ - tibialis anterior (7\%), peroneus longus (4\%) and vastus lateralis (4\%)- and a decreasing - Gmed (4\%) and Gmax (10\%)

292 - suggested that patients with CAI could be affected the neural activation pathways.(5)

\section{Clinical applications}

The results of the present study do not provide a gold standard approach for CAI patients. Thus, these findings aim to provide novel scientific evidence to the clinicians and researchers suggesting that DN technique could be effective in conjunction with the manual therapy or exercise programs in individuals with CAI. Nevertheless, future 
300 Some limitations should be acknowledged in the present study. First, baseline significant differences were found between groups at baseline for ATL-PPT and VAS, therefore the results of the present study for these variables might be biased. Second, for the YBT only one measure was taken for each variable. Third, an electromyographic evaluation did not carried out in order to evaluate the muscle activity of the extrinsic foot and the Gmed muscle. At last, the DN treatment was not observed in the medium or long term. Further research is recommended in order to evaluate the muscle activity, temperature of the MTrP or with a larger follow ups in patients with CAI.

\section{Conclusions}

The findings of the present study suggested that the DN application in the most hyperalgesic MTrP of the Gmed muscle may increase the anterior and medial dynamic balance, ankle dorsiflexion ROM and PPT-ATL at short-term in individuals with CAI. Pain intensity benefits were reported in both groups. Future studies should consider DN as a possible intervention in conjuction with a physical therapy program for individuals with CAI. Further research is still needed to improve the knowledge about the pain perception mechanisms developed in subjects with musculoskeletal disorders with invasive physiotherapy approaches.

\section{References}

321 1. Feger MA, Glaviano NR, Donovan L, Hart JM, Saliba SA, Park JS, et al. Current Trends in the Management of Lateral Ankle Sprain in the United States. Clin J Sport Med Off J Can Acad Sport Med. 2017 Mar;27(2):145-52.

2. Gribble PA, Bleakley CM, Caulfield BM, Docherty CL, Fourchet F, Fong DT-P, et al. 2016 consensus statement of the International Ankle Consortium: prevalence, impact and long-term consequences of lateral ankle sprains. $\mathrm{Br} \mathrm{J}$ Sports Med. 2016 Dec;50(24):1493-5. 
328 3. Kim H, Son SJ, Seeley MK, Hopkins JT. Altered Movement Biomechanics in Chronic Ankle Instability, Coper, and Control Groups: Energy Absorption and Distribution Implications. J Athl Train. 2019 Jun;54(6):708-17.

4. Nabb MTM, Kimber L, Haines A, McCourt C. Does regular massage from late pregnancy to birth decrease maternal pain perception during labour and birth?--A feasibility study to investigate a programme of massage, controlled breathing and visualization, from 36 weeks of pregnancy until birth. Complement Ther Clin Pract. 2006 Aug;12(3):222-31.

5. Son SJ, Kim H, Seeley MK, Hopkins JT. Altered Walking Neuromechanics in Patients With Chronic Ankle Instability. J Athl Train. 2019 Jun;54(6):684-97.

6. Dingenen B, Deschamps K, Delchambre F, Van Peer E, Staes FF, Matricali GA. Effect of taping on multi-segmental foot kinematic patterns during walking in persons with chronic ankle instability. J Sci Med Sport. 2017 Sep;20(9):835-40.

7. Chinn L, Dicharry J, Hertel J. Ankle kinematics of individuals with chronic ankle instability while walking and jogging on a treadmill in shoes. Phys Ther Sport. 2013 Nov;14(4):232-9.

8. Hopkins JT, Coglianese M, Glasgow P, Reese S, Seeley MK. Alterations in evertor/invertor muscle activation and center of pressure trajectory in participants with functional ankle instability. J Electromyogr Kinesiol. 2012 Apr;22(2):2805.

9. Koldenhoven RM, Feger MA, Fraser JJ, Saliba S, Hertel J. Surface electromyography and plantar pressure during walking in young adults with chronic ankle instability. Knee Surg Sports Traumatol Arthrosc. 2016 Apr;24(4):1060-70.

10. Delahunt E, Monaghan K, Caulfield B. Altered neuromuscular control and ankle joint kinematics during walking in subjects with functional instability of the ankle joint. Am J Sports Med. 2006 Dec;34(12):1970-6.

11. Delahunt E, Monaghan K, Caulfield B. Changes in lower limb kinematics, kinetics, and muscle activity in subjects with functional instability of the ankle joint during a single leg drop jump. J Orthop Res. 2006 Oct;24(10):1991-2000.

12. Hale SA, Fergus A, Axmacher R, Kiser K. Bilateral improvements in lower extremity function after unilateral balance training in individuals with chronic ankle instability. J Athl Train. 2014;49(2):181-91.

13. Van Deun S, Staes FF, Stappaerts KH, Janssens L, Levin O, Peers KKH. 
Relationship of chronic ankle instability to muscle activation patterns during the transition from double-leg to single-leg stance. Am J Sports Med. 2007 Feb;35(2):274-81.

14. Webster KA, Gribble PA. A comparison of electromyography of gluteus medius and maximus in subjects with and without chronic ankle instability during two functional exercises. Phys Ther Sport. 2013 Feb;14(1):17-22.

15. Gribble PA, Hertel J, Plisky P. Using the Star Excursion Balance Test to assess dynamic postural-control deficits and outcomes in lower extremity injury: a literature and systematic review. J Athl Train. 2012;47(3):339-57.

16. Plisky PJ, Gorman PP, Butler RJ, Kiesel KB, Underwood FB, Elkins B. The reliability of an instrumented device for measuring components of the star excursion balance test. N Am J Sports Phys Ther. 2009 May;4(2):92-9.

17. Bulow A, Anderson JE, Leiter JR, MacDonald PB, Peeler J. The modified star excursion balance and Y-balance test results differ when assessing physically active healthy adolescent females. Int J Sports Phys Ther . 2019 Apr;14(2):192203.

18. Ayotte NW, Stetts DM, Keenan G, Greenway EH. Electromyographical analysis of selected lower extremity muscles during 5 unilateral weight-bearing exercises. J Orthop Sports Phys Ther. 2007 Feb;37(2):48-55.

19. Jaber H, Lohman E, Daher N, Bains G, Nagaraj A, Mayekar P, et al. Neuromuscular control of ankle and hip during performance of the star excursion balance test in subjects with and without chronic ankle instability. PLoS One. 2018;13(8):e0201479.

20. Huguenin L, Brukner PD, McCrory P, Smith P, Wajswelner H, Bennell K. Effect of dry needling of gluteal muscles on straight leg raise: a randomised, placebo controlled, double blind trial. Br J Sports Med. 2005 Feb;39(2):84-90.

21. Shah JP, Thaker N, Heimur J, Aredo J V, Sikdar S, Gerber L. Myofascial Trigger Points Then and Now: A Historical and Scientific Perspective. PM R. 2015 Jul;7(7):746-61.

22. Dunning J, Butts R, Mourad F, Young I, Flannagan S, Perreault T. Dry needling: a literature review with implications for clinical practice guidelines. Phys Ther Rev. 2014 Aug;19(4):252-65.

23. Salom-Moreno J, Ayuso-Casado B, Tamaral-Costa B, Sánchez-Milá Z, Fernández-de-Las-Peñas C, Alburquerque-Sendín F. Trigger Point Dry Needling 
and Proprioceptive Exercises for the Management of Chronic Ankle Instability: A Randomized Clinical Trial. Evid Based Complement Alternat Med. 2015/04/30. 2015;2015:790209.

24. Hu H-T, Gao H, Ma R-J, Zhao X-F, Tian H-F, Li L. Is dry needling effective for low back pain?: A systematic review and PRISMA-compliant meta-analysis. Medicine (Baltimore). 2018 Jun;97(26):e11225.

25. Gattie E, Cleland JA, Snodgrass S. The Effectiveness of Trigger Point Dry Needling for Musculoskeletal Conditions by Physical Therapists: A Systematic Review and Meta-analysis. J Orthop Sports Phys Ther. 2017 Mar;47(3):133-49.

26. Martin-Rodriguez A, Saez-Olmo E, Pecos-Martin D, Calvo-Lobo C. Effects of dry needling in the sternocleidomastoid muscle on cervical motor control in patients with neck pain: a randomised clinical trial. Acupunct Med. 2019 Jun;37(3):151-63.

27. Sánchez-Mila Z, Salom-Moreno J, Fernández-de-Las-Peñas C. Effects of dry needling on post-stroke spasticity, motor function and stability limits: a randomised clinical trial. Acupunct Med. 2018/07/09. 2018 Dec;36(6):358-66.

28. Welch V, Jull J, Petkovic J, Armstrong R, Boyer Y, Cuervo LG, et al. Protocol for the development of a CONSORT-equity guideline to improve reporting of health equity in randomized trials. Implement Sci. 2015 Oct;10:146.

29. Gribble PA, Delahunt E, Bleakley C, Caulfield B, Docherty CL, Fourchet F, et al. Selection criteria for patients with chronic ankle instability in controlled research: a position statement of the International Ankle Consortium. J Orthop Sports Phys Ther. 2013 Aug;43(8):585-91.

30. Calvo-Lobo C, Pacheco-da-Costa S, Martínez-Martínez J, Rodríguez-Sanz D, Cuesta-Álvaro P, López-López D. Dry Needling on the Infraspinatus Latent and Active Myofascial Trigger Points in Older Adults With Nonspecific Shoulder Pain: A Randomized Clinical Trial. J Geriatr Phys Ther. 2018;41(1):1-13.

31. Chou L-W, Hsieh Y-L, Kuan T-S, Hong C-Z. Needling therapy for myofascial pain: recommended technique with multiple rapid needle insertion. BioMedicine. 2014;4:13.

32. Pecos-Martin D, Montanez-Aguilera FJ, Gallego-Izquierdo T, Urraca-Gesto A, Gomez-Conesa A, Romero-Franco N, et al. Effectiveness of dry needling on the lower trapezius in patients with mechanical neck pain: a randomized controlled trial. Arch Phys Med Rehabil. 2015 May;96(5):775-81. 
33. Tejera-Falcón E, Toledo-Martel NDC, Sosa-Medina FM, Santana-González F, Quintana-de la Fe MDP, Gallego-Izquierdo T, et al. Dry needling in a manual physiotherapy and therapeutic exercise protocol for patients with chronic mechanical shoulder pain of unspecific origin: a protocol for a randomized control trial. BMC Musculoskelet Disord. 2017 Sep 18;18(1):400.

34. Neziri AY, Scaramozzino P, Andersen OK, Dickenson AH, Arendt-Nielsen L, Curatolo M. Reference values of mechanical and thermal pain tests in a pain-free population. Eur J Pain. 2011 Apr;15(4):376-83.

35. Cheatham SW, Kolber MJ, Mokha GM, Hanney WJ. Concurrent validation of a pressure pain threshold scale for individuals with myofascial pain syndrome and fibromyalgia. J Man Manip Ther. 2017/07/09. 2018 Feb;26(1):25-35.

36. Fischer AA. Algometry in Diagnosis of Musculoskeletal Pain and Evaluation of Treatment Outcome: An Update. J Musculoskelet Pain. 1998 Jan 1;6(1):5-32.

37. Gribble PA, Hertel J. Considerations for Normalizing Measures of the Star Excursion Balance Test. Meas Phys Educ Exerc Sci. 2003 Jun 1;7(2):89-100.

38. Konor MM, Morton S, Eckerson JM, Grindstaff TL. Reliability of three measures of ankle dorsiflexion range of motion. Int J Sports Phys Ther. 2012 Jun;7(3):279-87.

39. Fernandez-Carnero J, La Touche R, Ortega-Santiago R, Galan-del-Rio F, Pesquera J, Ge H-Y, et al. Short-term effects of dry needling of active myofascial trigger points in the masseter muscle in patients with temporomandibular disorders. J Orofac Pain. 2010;24(1):106-12.

40. Itoh K, Saito S, Sahara S, Naitoh Y, Imai K, Kitakoji H. Randomized trial of trigger point acupuncture treatment for chronic shoulder pain: a preliminary study. J Acupunct Meridian Stud. 2014 Apr;7(2):59-64.

41. Zheng Y, Shi D, Wu X, Gu M, Ai Z, Tang K, et al. Ultrasound-Guided Miniscalpel-Needle Release versus Dry Needling for Chronic Neck Pain: A Randomized Controlled Trial. Evid Based Complement Alternat Med. 2014;2014:235817.

42. Mayoral O, Salvat I, Martin MT, Martin S, Santiago J, Cotarelo J, et al. Efficacy of myofascial trigger point dry needling in the prevention of pain after total knee arthroplasty: a randomized, double-blinded, placebo-controlled trial. Evid Based Complement Alternat Med. 2013;2013:694941.

43. Cotchett MP, Munteanu SE, Landorf KB. Effectiveness of trigger point dry 
needling for plantar heel pain: a randomized controlled trial. Phys Ther. 2014 Aug;94(8):1083-94.

44. Liu L, Huang Q-M, Liu Q-G, Ye G, Bo C-Z, Chen M-J, et al. Effectiveness of dry needling for myofascial trigger points associated with neck and shoulder pain: a systematic review and meta-analysis. Arch Phys Med Rehabil. 2015 May;96(5):944-55.

45. Llamas-Ramos R, Pecos-Martin D, Gallego-Izquierdo T, Llamas-Ramos I, PlazaManzano G, Ortega-Santiago R, et al. Comparison of the short-term outcomes between trigger point dry needling and trigger point manual therapy for the management of chronic mechanical neck pain: a randomized clinical trial. J Orthop Sports Phys Ther. 2014 Nov;44(11):852-61.

46. Kamali F, Sinaei E, Morovati M. Comparison of Upper Trapezius and Infraspinatus Myofascial Trigger Point Therapy by Dry Needling in Overhead Athletes With Unilateral Shoulder Impingement Syndrome. J Sport Rehabil. 2019 Mar;28(3):243-9.

47. Abbaszadeh-Amirdehi M, Ansari NN, Naghdi S, Olyaei G, Nourbakhsh MR. Neurophysiological and clinical effects of dry needling in patients with upper trapezius myofascial trigger points. J Bodyw Mov Ther. 2017 Jan;21(1):48-52.

48. Onat SS, Polat CS, Bicer S, Sahin Z, Tasoglu O. Effect of Dry Needling Injection and Kinesiotaping on Pain and Quality of Life in Patients with Mechanical Neck Pain. Pain Physician. 2019 Nov;22(6):583-9.

49. Mendigutia-Gomez A, Martin-Hernandez C, Salom-Moreno J, Fernandez-deLas-Penas C. Effect of Dry Needling on Spasticity, Shoulder Range of Motion, and Pressure Pain Sensitivity in Patients With Stroke: A Crossover Study. J Manipulative Physiol Ther. 2016 Jun;39(5):348-58.

50. Benito-de-Pedro M, Becerro-de-Bengoa-Vallejo R, Losa-Iglesias ME, Rodríguez-Sanz D, López-López D, Cosín-Matamoros J, et al. Effectiveness between Dry Needling and Ischemic Compression in the Triceps Surae Latent Myofascial Trigger Points of Triathletes on Pressure Pain Threshold and Thermography: A Single Blinded Randomized Clinical Trial. J Clin Med. 2019 Oct 5;8(10):1632.

51. Lake AD, Myers H, Aefsky B, Butler R. Immediate and short term effect of dry needling on triceps suare range of motion and functional movement: a randomized trial. Int J Sports Phys Ther [Internet]. 2018 Apr;13(2):185-95. 
Available from: https://pubmed.ncbi.nlm.nih.gov/30090677

499

52. MacKinnon CD, Winter DA. Control of whole body balance in the frontal plane during human walking. J Biomech. 1993 Jun;26(6):633-44.

501

53. Hall ML, Mackie AC, Ribeiro DC. Effects of dry needling trigger point therapy

502 in the shoulder region on patients with upper extremity pain and dysfunction: a

503 systematic review with meta-analysis. Physiotherapy. 2018 Jun;104(2):167-77.

504

54. Dunning J, Butts R, Henry N, Mourad F, Brannon A, Rodriguez H, et al.

505 Electrical dry needling as an adjunct to exercise, manual therapy and ultrasound

506 for plantar fasciitis: A multi-center randomized clinical trial. PLoS One.

507 2018;13(10):e0205405.

508

509

\section{$510 \quad$ Figure legends.}

511 Figure 1. Flow chart diagram.

512 Figure 2. Comparison between intervention and control group measurements for YBT, 513 PPT and VAS variables. * Significant differences between groups (treatment $\mathrm{x}$ 514 time).

515

516 
Table 1. Sociodemographic data and CAIT scale of the sample

\section{Total sample $(n=40) \quad$ Intervention}

$P$-value Cases vs

Data $\quad(n=20) \quad$ Controls $(n=20) \quad$ Controls

\begin{tabular}{|c|c|c|c|c|}
\hline Age, $y$ & $32.50 \pm 6.78$ & $33.80 \pm 6.77$ & $31.20 \pm 6.70$ & .230 \\
\hline Weight, kg & $68.52 \pm 12.02$ & $70.60 \pm 13.27$ & $66.85 \pm 10.65$ & .331 \\
\hline Height, m & $1.69 \pm 0.18$ & $1.66 \pm 0.25$ & $1.73 \pm 0.07$ & .220 \\
\hline BMI, $\mathrm{kg} / \mathrm{m}^{2}$ & $22.81 \pm 1.35$ & $24.61 \pm 1.05$ & $25.36 \pm 1.47$ & .350 \\
\hline CAIT & $22.30 \pm 2.24$ & $22.60 \pm 2.03$ & $22.00 \pm 2.44$ & .405 \\
\hline
\end{tabular}

Abbreviations: BMI, body mass index; CAIT, Cumberland ankle instability tool. 
Table 2. SEB, ROM, VAS and PPT intrasubject effects.

\section{Intrasubject Effects}

\begin{tabular}{|c|c|c|c|c|}
\hline Measure & $\begin{array}{l}\text { Intervention } \\
\mathrm{n}=20\end{array}$ & Control $n=20$ & $\frac{\text { Time value }}{\mathrm{F} ; \mathrm{P}\left(\mathrm{Eta}^{2}\right)}$ & $\frac{\text { Treatment X Time }}{\mathrm{F} ; \mathrm{P}\left(\mathrm{Eta}^{2}\right)}$ \\
\hline YBT anterior & & & $\mathrm{F}=22.272 ; \mathrm{P}=.001(0.370)$ & $\mathrm{F}=7.425 ; \mathrm{P}=.001(0.163)$ \\
\hline Baseline & $94.06 \pm 24.78$ & $97.56 \pm 8.96$ & & \\
\hline Post-test & $99.28 \pm 24.56$ & $98.52 \pm 8.73$ & & \\
\hline 1-week & $100.68 \pm 23.58$ & $99.53 \pm 8.95$ & & \\
\hline YBT medial & & & $\mathrm{F}=28.391 ; \mathrm{P}=.001(0.428)$ & $\begin{array}{l}F=15.075 ; P=.001 \\
(0.284)\end{array}$ \\
\hline Baseline & $131.06 \pm 16.41$ & $134.50 \pm 15.92$ & & \\
\hline Post-test & $138.80 \pm 16.46$ & $135.67 \pm 15.30$ & & \\
\hline 1-week & $140.96 \pm 15.43$ & $136.07 \pm 14.98$ & & \\
\hline YBT lateral & & & $\mathrm{F}=9.650 ; \mathrm{P}=.001(0.203)$ & $(0.079)$ \\
\hline Baseline & $120.83 \pm 21.24$ & $126.12 \pm 17.21$ & & \\
\hline Post-test & $126.08 \pm 21.77$ & $127.20 \pm 16.96$ & & \\
\hline 1-week & $126.97 \pm 19.26$ & $127.96 \pm 17.11$ & & \\
\hline 1-week & $77.0 \pm 4.8$ & $73.2 \pm 6.2$ & & \\
\hline Dorsiflexion & & & & \\
\hline ROM & & & $\mathrm{F}=8.436 ; \mathrm{P}=.001(0.182)$ & $\mathrm{F}=7.728 \mathrm{P}=.001(0.169)$ \\
\hline Baseline & $149.15 \pm 11.89$ & $133.40 \pm 13.77$ & & \\
\hline Post-test & $152.85 \pm 12.23$ & $133.55 \pm 13.53$ & & \\
\hline 1-week & $152.25 \pm 11.03$ & $133.35 \pm 13.56$ & & \\
\hline ATL PPT & & & $\mathrm{F}=40.613 ; \mathrm{P}=.001(0.517)$ & $\begin{array}{l}F=9.372 ; P=.002 \\
(0.198)\end{array}$ \\
\hline Baseline & $3.21 \pm 0.82$ & $3.91 \pm 0.89$ & & \\
\hline Post-test & $3.92 \pm 0.93$ & $4.11 \pm 0.98$ & & \\
\hline 1-week & $4.04 \pm 0.96$ & $4.23 \pm 1.01$ & & \\
\hline Gmed PPT & & & $\mathrm{F}=6.559 ; \mathrm{P}=.009(0.147)$ & $\begin{array}{l}F=1.037 ; P=.332 \\
(0.027)\end{array}$ \\
\hline Baseline & $5.46 \pm 2.16$ & $5.65 \pm 1.24$ & & \\
\hline Post-test & $5.79 \pm 2.33$ & $5.77 \pm 1.23$ & & \\
\hline 1-week & $5.91 \pm 2.29$ & $5.86 \pm 1.18$ & & \\
\hline
\end{tabular}

Values are mean \pm SD unless otherwise indicated.

Abbreviature: ATL, anterior talofibular ligament; Gmed, gluteus medius; PPT, pain pressure threshold; ROM, range of motion; SEB, star excursion balance; YBT, Ybalance test. 


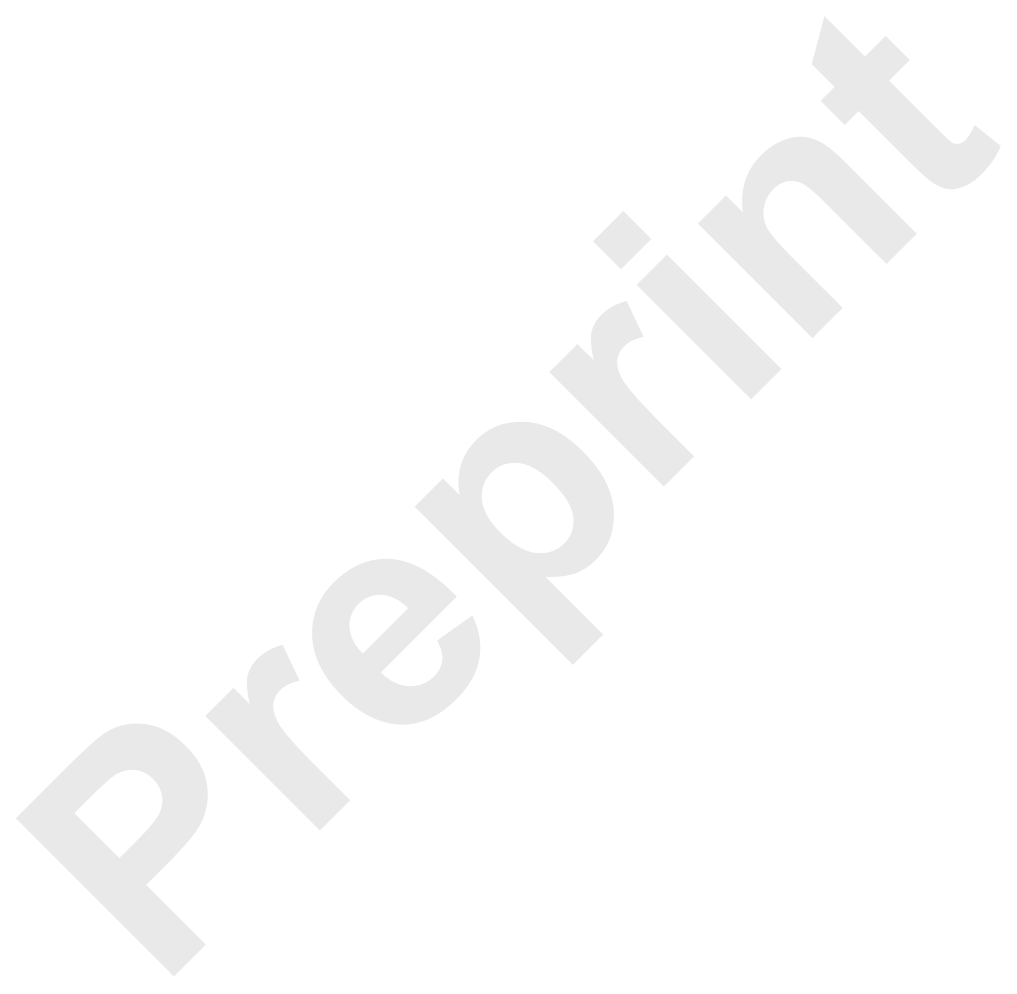




\section{CONSORT \\ TRANSPARENT REPORTING Of TRIALS}

\section{CONSORT Flow Diagram}

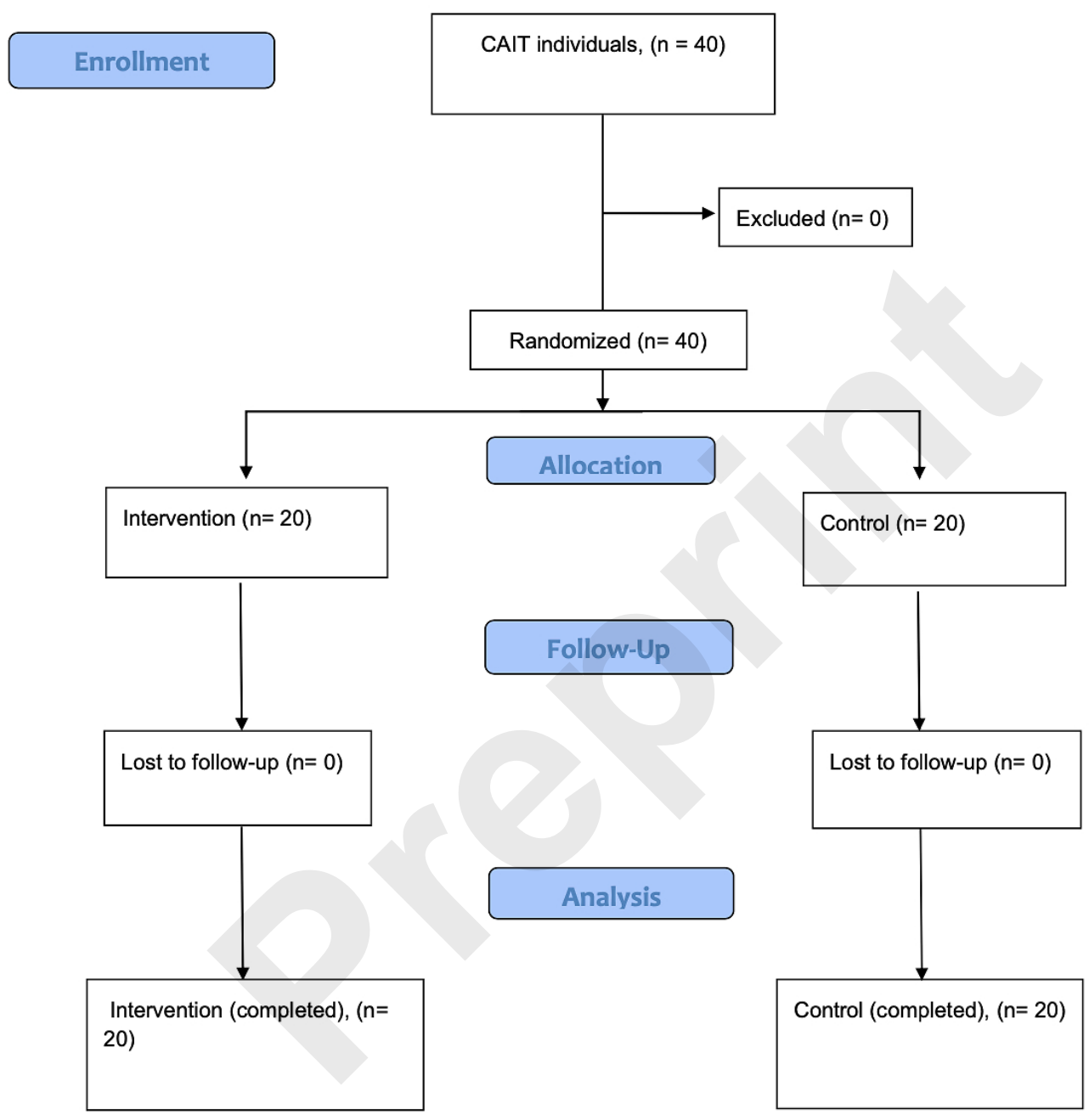

Flow chart diagram 

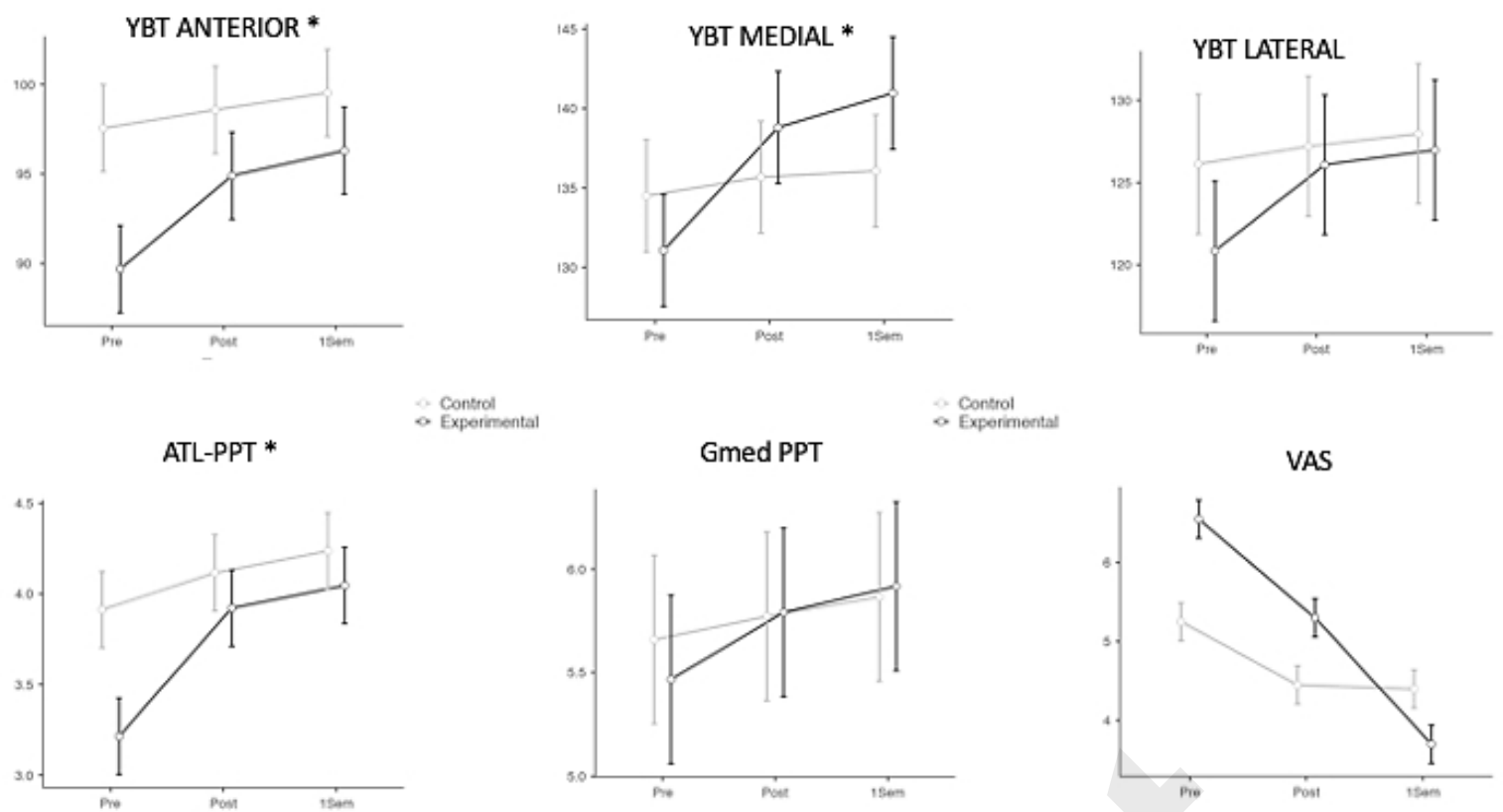

Control
- Exporimenta

Gmed PPT

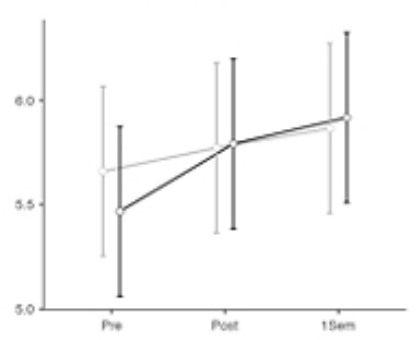

VAS

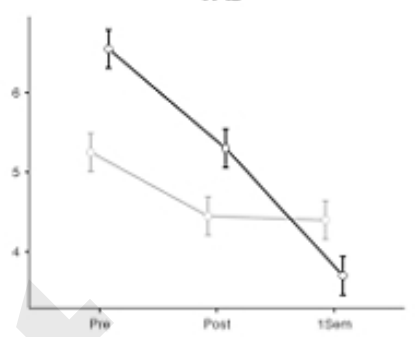

Comparison between intervention and control group measurements for YBT, PPT and VAS variables. * Significant differences between groups (treatment $x$ time). 